\title{
GROMOV'S COMPACTNESS THEOREM FOR PSEUDO HOLOMORPHIC CURVES
}

\author{
RUGANG YE
}

\begin{abstract}
We give a complete proof for Gromov's compactness theorem for pseudo holomorphic curves both in the case of closed curves and curves with boundary.
\end{abstract}

\section{INTRODUCTION}

In $1985 \mathrm{M}$. Gromov invented the beautiful theory of pseudo holomorphic curves and made it a powerful tool in symplectic geometry [Gro]. On a symplectic manifold he considered almost complex structures which are "compatible" with or more generally, "tamed" by the symplectic structure and studied the space of "pseudo" holomorphic curves defined in terms of one such almost complex structure. The "moduli space" of pseudo holomorphic curves then contains invariants of the symplectic structure. In spirit, this can be compared with gauge theory on 4-manifolds, where the moduli space of anti-self-dual connections on a principal bundle over a given 4-manifold contains topological invariants of the manifold. The first question one asks about a moduli space is whether it is compact. If it is not compact, then one would like to understand its compactification. The total moduli space of pseudo holomorphic curves is too big, and one needs to restrict to fixed topological types and impose an area bound. But even under these restrictions, in general one still cannot expect compactness due to three reasons. First, holomorphic spheres may split or bubble off a sequence of holomorphic curves (we abandon the adjective "pseudo" henceforth). Second, the underlying conformal structures of a sequence of holomorphic curves may degenerate. Third, bubbling-off of holomorphic disks may also occur if one is dealing with holomorphic curves with boundary. To deal with these degenerations, Gromov introduced the notion of "cusp-curves", which are basically holomorphic curves joint at isolated points. (Holomorphic curves are included in this concept.) Gromov's compactness theorem says that a sequence of holomorphic curves of a fixed topological type and uniformly bounded area converges to a cusp-curve. The convergence is basically $C^{\infty}$-convergence away from isolated singular points plus area convergence. Note that this goes beyond symplectic geometry and applies to arbitrary almost complex manifolds. But in case of a tame almost complex structure a big advantage is at hand, namely a uniform area bound for closed holomorphic curves holds whenever

Received by the editors April 19, 1992.

1991 Mathematics Subject Classification. Primary 53C15; Secondary 35D10. 
their homology class is fixed. Hence Gromov's compactness theorem yields for a tame almost complex manifold a natural compactification of the moduli space of closed holomorphic curves of a fixed topological type and a fixed homology class. It should be emphasized that without a good compactification the (generally noncompact) moduli space is virtually useless, because all information can be carried away along divergence. Of course, one even hopes to obtain compactness of some interesting components of the moduli space themselves under reasonable assumptions. Such compactness results are in fact consequences of Gromov's compactness theorem. For example, one derives compactness of the space of holomorphic disks under suitable conditions. This has important applications in the theory of filling by holomorphic disks as developed by Gromov, Eliashberg, and others [Gro, El]. Note that in these compactness results the taming condition is needed to control area. On a general almost complex manifold, it is not clear yet what natural assumptions imply area bounds on holomorphic curves. But applications of Gromov's theory are promising and we emphasize the generality of Gromov's compactness theorem.

Gromov proved his compactness theorem for closed holomorphic curves by a geometric argument based on Schwarz' lemma and an isoperimetric inequality. More details of this argument has been written up by P. Pansu [Pan]. To deal with holomorphic curves with boundary, Gromov suggested to make a reflection across the (totally real) boundary manifold in which the boundaries of the holomorphic curves lie and thereby to reduce to the boundaryless case. If the ambient almost complex manifold is integrable near the boundary manifold and the boundary manifold is real analytic, the reflection argument indeed works and has been carried out by Pansu [Pan]. In general, the reflection argument encounters serious difficulties and has not been carried out so far.

In [Wo] J. Wolfson took a different point of view and followed the covering and rescaling scheme of Sacks-Uhlenbeck for harmonic mappings [Sa-U]. SacksUhlenbeck's approach is based on an $\varepsilon$-regularity theorem (i.e., a gradient estimate under the small energy assumption) and removal of isolated singularities. To obtain an $\varepsilon$-regularity theorem for holomorphic curves, Wolfson derived a Bochner formula for energy density and applied an a priori estimate argument of R. Schoen for harmonic mappings [Sc]. Gromov and Pansu's result on removal of isolated singularities was then applied. This way Wolfson recovered a part of Gromov's compactness theorem. Namely he proved convergence of closed holomorphic curves to cusp-curves, but his convergence is weaker than the convergence in Gromov's theorem, in particular area convergence is not recovered. (Thus some area is lost in the limit. In case of varying conformal structures, area might be lost completely.) In a recent paper [Par-Wo], T. Parker and J. Wolfson considered the issue of full convergence for closed holomorphic curves of a fixed conformal structure. But their argument is wrong. (More recently, they adopted the idea of using the isoperimetric inequality in the present paper and revised their paper.) It should be emphasized that area convergence in Gromov convergence is extremely important. In particular, homology can be lost if area loss occurs.

As about extension to holomorphic curves with boundary, we note that the Bochner formula argument does not automatically applies to the boundary value problem because a corresponding boundary condition for the third order Bochner equation is missing. The more crucial problem is however removal 
of isolated singularities at boundary. We would like to mention that Y. G. Oh [Oh] has proven removal of boundary singularities for the special case where one has an almost complex structure which is compatible with a given symplectic structure and a Lagrangian boundary manifold. Oh's method depends heavily on both the underlying symplectic structure and the Lagrangian condition.

In the present paper we adopt a new approach to Gromov's compactness theorem.

We resolve the problem of holomorphic curves with boundary and also present a complete treatment of closed holomorphic curves. Thus we prove Gromov's compactness theorem in its full generality both for closed holomorphic curves and holomorphic curves with boundary. We follow Sacks-Uhlenbeck's scheme, but removal of isolated singularities and $\varepsilon$-regularity will be derived by a new argument. In fact, both results will be consequences of a regularity theorem for weakly holomorphic curves. The basic idea is that for a suitably chosen Riemannian metric, holomorphic curves are very similar to minimal surfaces. First, they satisfy an equation of harmonic mapping type. In local coordinates this equation consists of a linear Laplacian term as the top order term and a term which is quadratic in the gradient. Second, they are conformal, and this is very important. Our observation is that a regularity argument of $\mathbf{M}$. Grüter for minimal surfaces [Grü] can be carried over to yield continuity of weakly holomorphic curves. Gradient estimates will then be derived by a variational argument.

Geometrically, the above two facts imply that the unbranched part of a holomorphic curve has bounded mean curvature vector. We employ this to obtain an isoperimetric inequality for arbitrary domains of a holomorphic curve. Previously, such an inequality was only known for holomorphic curves in a tame almost complex manifold [Pan, Par-Wo].

Once the interior regularity theorem and hence $\varepsilon$-regularity and removal of interior singularities are at hand, we can apply Sacks-Uhlenbeck's covering and rescaling scheme to deal with convergence of closed holomorphic curves. Thus we obtain, e.g., holomorphic spheres or bubbles at blow-up points.

In order to obtain the full Gromov convergence, we design an iteration procedure to capture all bubbles which splitt off bubbles. The real problem here is how to control "bubble necks", which are annulas regions between bubbles or between a bubble and the converging part of a holomorphic curve under inspection. One needs to know whether these necks are actually also bubbles and if not, to show that their total area tends to zero. We shall employ length of circles to detect bubbles, therewith achieving the first task. The desired area estimate will be derived from the isoperimetric inequality mentioned above. Pinching necks which arise from degeneration of conformal structures are treated in a similar way.

We would like to insert a general remark here. There are a few well-known geometric variational problems which possess conformal invariance, such as Yang-Mills fields and harmonic mappings (both in appropriate dimensions.) Bubbling is a characteristic phenomenon in these problems. It is very important to rule out "energy loss" when bubbling occurs. So far there is no general argument for ruling out energy loss which applies to all those conformally invariant problems. Conformal scaling is a routine argument here; it is important but does not solve the problem by itself. We believe that additional geomet- 
ric structures have to be used. Our way of dealing with holomorphic curves depends on the isoperimetric inequality.

Next we come to holomorphic curves with boundary. Here we make analogy with minimal surfaces which possess a free boundary. For a given almost complex manifold and a given totally real submanifold $N$ we can choose a suitable metric to make the analogy of holomorphic curves with minimal surfaces as discussed above and to simultaneously render $N$ "pseudo-Lagrangian". The pseudo-Lagrangian condition implies that holomorphic curves with boundary on $N$ have to meet $N$ orthogonally. This enables us to carry over the arguments of Grüter-Hildebrandt-Nitsche [Grü-Hi-Ni] and J. Jost [Jo1] for minimal surfaces with free boundary to obtain the desired boundary regularity theorem. $\left(C^{1}\right.$ estimates again are derived by a variational argument.) The above iteration procedure with some modifications is then applied.

Now let us state Gromov's compactness theorem in full details. Let $(M, J)$ be a compact almost complex manifold of real dimension $2 n$, where $J$ denotes an almost complex structure, and $S$ be a Riemann surface with complex structure $j$. A smooth map $f:(S, j) \rightarrow(M, J)$ is called a pseudo holomorphic curve (or a $J$-holomorphic curve or simply a holomorphic curve) if $d f \circ j=J \circ d f$. A totally real submanifold of $(M, J)$ is a submanifold $N$ of dimension $n$ such that $J(T N) \cap T N=$ the zero section. We fix a Riemannian metric $g$ on $M$.

Definition 0.1. A cusp-curve in $(M, J)$ is a disjoint union of finitely many Riemann surfaces $S_{\alpha}$, together with an identification of a finite number of points (called "nodes") and a holomorphic curve $f: \bigcup_{\alpha} S_{\alpha} \rightarrow M$ compatible with the identification. Boundary nodes are identified with boundary nodes only.

$S=\bigcup_{\alpha} S_{\alpha}$ is called a Riemann surface with nodes. We set genus $(S)=$ $\sum_{\alpha} \operatorname{genus}\left(S_{\alpha}\right), \operatorname{conn}(S)=\sum_{\alpha} \operatorname{conn}\left(S_{\alpha}\right)$, where conn (connectivity) means the number of boundary components. The homology class $[f]$ of $f$ is defined to be $\sum_{\alpha}\left[f \mid S_{\alpha}\right]$.

A continuous surjective map $\varphi: S \rightarrow S^{\prime}$ between two Riemann surfaces with nodes is called a node map, if the following hold: (i) for each node $x$ of $S^{\prime}$, $\varphi^{-1}(x)$ is either a node, a simple closed curve in the interior which is disjoint from nodes or a simple arc which is disjoint from nodes and has exactly its endpoints on the boundary, (ii) $\varphi$ is a diffeomorphism away from the curves which are preimage of nodes. A diffeomorphism is a trivial node map. Thus, topologically $S^{\prime}$ is obtained from $S$ by collapsing certain simple curves to points, if a nontrivial node map from $S$ to $S^{\prime}$ exists.

Note $\operatorname{genus}\left(S^{\prime}\right) \leq \operatorname{genus}(S)$ and $\operatorname{conn}\left(S^{\prime}\right) \geq \operatorname{conn}(S)$.

Definition 0.2 (The $C^{k}$ topology on the space of compact cusp-curves). Let $f: S \rightarrow M$ be a compact cusp-curve ("compact" means that $S$ is compact). For $\varepsilon>0$, a metric on $S$ and a neighborhood $U$ of the nodes of $S$, a neighborhood $F$ of $f$ is defined as follows. A compact cusp-curve $\tilde{f}: \tilde{S} \rightarrow M$ belongs to $F$ if

(i) there is a node map $\varphi: S \rightarrow \tilde{S}$,

(ii) $\left\|f-\tilde{f} \circ \varphi^{-1}\right\|_{C^{k}}<\varepsilon$ on $S \backslash U$, 
(iii) $\left\|j-\varphi^{*} \tilde{j}\right\|_{C^{k}}<\varepsilon$ on $S \backslash U$,

(iv) $|\operatorname{area}(f)-\operatorname{area}(\tilde{f})|<\varepsilon$,

where $j, \tilde{j}$ denote the complex structures of $S$ and $\tilde{S}$ respectively, and the norms and area are defined in terms of the given metrics on $M$ and $S$.

Remark 1. It turns out that for closed cusp-curves and cusp-curves with boundary on totally real submanifolds all $C^{k}$ topologies are equivalent.

Remark 2. It is easy to see that $C^{k}$ topology preserves homology, i.e., if $f_{l} \rightarrow f$, then $\left[f_{l}\right]=[f]$ for large $l$. (This is immediately clear in our proof of Gromov convergence.) This fact is important in applications. Suitably defined homotopy is also preserved.

Let $\mathscr{C}$ denote the space of compact cusp-curves in $(M, J)$ and $\mathscr{F} \subset \mathscr{C}$ the space of closed holomorphic curves. The groups of conformal automorphisms act on $\mathscr{C}$, and we use ${ }^{*}$ to denote the quotient (projection). The bar on top will denote closure with respect to the $C^{k}$ topology for some $k$ (by the above remark this is independent of $k$ in the following situations).

Theorem 0.1 (Gromov's compactness theorem for closed pseudo holomorphic curves).

(i) For $m \in \mathbb{N} \cup\{0\}, a>0$, let $\mathscr{F}_{m, a}$ denote the subspace of $\mathscr{F}$ defined by the conditions genus $=m$ and area $\leq a$. For each $m$ and $a, \overline{\mathscr{F}}_{m, a}$ is (sequentially) compact.

(ii) Let $\left\{f_{l}\right\}$ be a sequence of nonconstant holomorphic curves in $\mathscr{T}_{m, a}$ for some $m, a$ such that $\lim f_{l}^{*} \in \overline{\mathscr{F}}_{m, a}^{*} \backslash \mathscr{F}_{m, a}^{*}$. Then at least one of the following happens: (1) the conformal structures of $f_{l}$ degenerate, (2) each $f \in \lim f_{l}^{*}$ consists of more than one nonconstant holomorphic curves.

Remark 1. One gets similar results if one allows $J$ to vary in a compact set. These results are needed in applications and can be considered as corollaries.

Remark 2. Our motivation for including the second part comes from [Mc].

Remark 3. The space $\mathscr{F}_{0, a}$ can never be compact because of the action of the Möbius group. Instead, one gets compactness of (components of) $\mathscr{F}_{0, a}^{*}$ in many situations, see [Mc].

Recall that $J$ is called a tame almost complex structure, if there is a simplectic structure $\omega$ on $M$ such that $\omega(\cdot, J)$ is positive definite. $\omega$ is called a taming form for $J$. By the way, if moreover $\omega(\cdot, J)$ is symmetric (equivalently, $\omega(J, J)=\omega)$, then we say that $J$ is symplectic, and call $\omega$ a compatible form for $J$ and $J$ a compatible almost complex structure for $\omega$. As mentioned before, taming property can be used to control area, whence we have

Corollary. If $(M, J)$ is tame, then $\overline{\mathscr{F}}_{m, \gamma}$ is compact for each $m, \gamma$, where $m$ indicates genus and $\gamma$ a homology class in $H_{2}(M, \mathbb{Z})$.

Next we consider a compact, totally real submanifold $N$ of $(M, J)$. Let $\mathscr{F}(N)$ be the space of compact holomorphic curves with boundary on $N$. For $m \in \mathbb{N} \cup\{0\}, m^{\prime} \in \mathbb{N}$ and $a>0$, let $\mathscr{F}(N)_{m, m^{\prime}, a}$ be the subspace of $\mathscr{F}(N)$ defined by the conditions genus $=m$, conn $=m^{\prime}$ and area $\leq a$. 
Theorem 0.2 (Gromov's compactness theorem for pseudo holomorphic curves with boundary).

(i) For each $m, m^{\prime}$, and $a, \overline{\mathscr{F}(N)}_{m, m^{\prime}, a}$ is compact.

(ii) Let $\left\{f_{l}\right\}$ be a sequence of nonconstant holomorphic curves in $\mathscr{F}(N)_{m, m^{\prime}, a}$ for some $m, a$ such that

$$
\lim f_{l}^{*} \in{\overline{\mathscr{F}(N)_{m, m^{\prime}, a}}} \mid \mathscr{F}(N)_{m, m^{\prime}, a} .
$$

Then at least one of the following happens: (1) the conformal structures of $f_{l}$ degenerate, (2) each $f \in \lim f_{l}^{*}$ consists of more than one nonconstant holomorphic curves.

Remark. Again, one can allow $J$ to vary in a compact set. One can also allow $N$ to vary in a compact set (of submanifolds).

We acknowledge stimulating conversations with $\mathrm{W}$. Klingenberg, Jr., T. Mrowka, J. D. Moore and Y. Eliashberg.

\section{INTERIOR REGULARITY OF HOLOMORPHIC CURVES}

Let $(M, J)$ be an almost complex manifold of real dimension $2 n$. We assume that $(M, J)$ has bounded geometry, i.e., there is a hermitian metric $g$ such that $(M, g)$ has nonzero injectivity radius and uniformly bounded sectional curvatures, and in addition $D J$ is also uniformly bounded, where $D$ denotes the Levi-Civita connection. (A Riemannian metric on $(M, J)$ is called a hermitian metric if $J$ is an isometry everywhere.) Such a metric will be called "preferred". We fix a preferred metric $g$. Note that compact almost complex manifolds have bounded geometry.

Definition 1.1. A weakly holomorphic curve in $(M, J)$ is an $H^{1,2}$ map $f$ from a compact Riemann surface $(S, j)$ into $(M, J)$ such that $J \circ d f=d f \circ j$ almost everywhere. (For a noncompact $M$, this definition depends on the choice of a hermitian metric on $M$ because of the $H^{1,2}$ requirement.)

Theorem 1.1. Weakly holomorphic curves are smooth in the interior. Moreover, for some positive constants $\delta$ and $C$ depending only on $J$ and $g$ the following holds. (The dependence of $C$ on $J, g$ is $C=C(\sup \|D J\|, i(M)$, sup $|K|)$, where $i(M)$ is the injectivity radius of $M$ and $K$ denotes sectional curvatures. The precise dependence can be read off the proof.)

Let $(S, j)$ be a compact Riemann surface together with its Poincaré metric $h$ and $f:(S, j) \rightarrow(M, J)$ a weakly holomorphic curve. Consider $w \in \stackrel{\circ}{S}$ and $0<R \leq \min \{1, \operatorname{inj}(w)\}$ where $\operatorname{inj}(w)$ is the injectivity radius at $w$. If $A(R):=\operatorname{area}\left(\left.f\right|_{B_{R}(w)}\right)<\delta$, where $B_{R}(w)$ denotes the (closed) geodesic ball of center $w$ and radius $R$, then

$$
\sup _{B_{R / 2}(w)}\|d f\|^{2} \leq C A(R) / R^{2} .
$$

Proof. It suffices to consider the situation as described for the a priori estimates. Let $\{u, v\}$ be a conformal coordinate system on $B_{R}(w)$ such that $j(\partial / \partial u)=$ $\partial / \partial v$. For the sake of achieving estimates, we actually choose $\{u, v\}$ to be stereographic coordinates centered at $p$ in case of a spherical $h$, euclidean coordinates in case of a flat $h$ and Poincaré coordinates centered at $p$ in case of a hyperbolic $h$. 
Then we have almost everywhere

$$
J\left(\frac{\partial f}{\partial u}\right)=\frac{\partial f}{\partial v}, \quad J\left(\frac{\partial f}{\partial v}\right)=-\frac{\partial f}{\partial u} .
$$

Hence for a $H^{1,2}$ vector field $X$ along $f$,

$$
J\left(\frac{\partial f}{\partial u}\right) \cdot \frac{\partial X}{\partial u}=\frac{\partial f}{\partial v} \cdot \frac{\partial X}{\partial u}, \quad J\left(\frac{\partial f}{\partial v}\right) \cdot \frac{\partial X}{\partial v}=-\frac{\partial f}{\partial u} \cdot \frac{\partial X}{\partial v},
$$

where differentiation of $X$ refers to the connection $D$. To proceed, we embed $(M, g)$ isometrically into a euclidean space. Since $f$ is a weak limit of smooth maps into the euclidean space, we can apply integration by parts to achieve

$$
\int_{B_{R}(w)} \frac{\partial f}{\partial v} \cdot \frac{\partial X}{\partial u}=\int_{B_{R}(w)} \frac{\partial f}{\partial u} \cdot \frac{\partial X}{\partial v},
$$

provided that $X$ has support contained in $B_{R}(w)$. Here the integration is with respect to the euclidean metric $d u^{2}+d v^{2}$. From (1.3) and (1.4) we then obtain the equation

$$
\int_{B_{R}(w)}\left(J\left(\frac{\partial f}{\partial u}\right) \cdot \frac{\partial X}{\partial u}+J\left(\frac{\partial f}{\partial v}\right) \cdot \frac{\partial X}{\partial v}\right)=0
$$

for $X$ with support contained in $\stackrel{\circ}{B}_{R}(w)$. This can be invariantly written as

$$
\int\left\langle J \circ d f, D_{J} X\right\rangle=0 \text {, }
$$

where the integration is with respect to $h$ and $X$ is assumed to have support in $\stackrel{\circ}{S}$. Because $R \leq 1$, we can replace $B_{R}(w)$ by the euclidean ball $D_{R_{1}}(w)$ for $R_{1}=a R$, where $a$ is a universal positive constant, and require $X$ to have support in $D_{R_{1}}(w)$. We are going to show first that $f$ is continuous.

Let $\rho_{0}=\frac{1}{8} \min (\pi / 2 \wedge, i(M))$, where $\wedge=\sup |K|$. Consider $z_{0} \in \stackrel{\circ}{D}_{R_{1}}(w)$ and $r \leq d\left(z_{0}, \partial D_{R_{1}}(w)\right)$ with $d$ denoting the euclidean distance. We want to estimate the oscillation of $f$ on the ball $D_{r}\left(z_{0}\right)$. Assume that the trace of $f$ on $\partial D_{r}\left(z_{0}\right)$ is continuous. Consider a Lebesgue point $z_{1} \in D_{r}\left(z_{0}\right)$ such that $d(r):=\inf _{z \in \partial D_{r}\left(z_{0}\right)} d\left(f(z), f\left(z_{1}\right)\right)$ is positive. We are going to control $d(r)$.

Fix a $\rho^{\prime} \in(0, d(r))$. We put $a=f\left(z_{1}\right)$ and consider an arbitrary $\rho \in$ $\left(0, \rho^{\prime}\right)$. Given $\varepsilon>0$, choose some $\varphi \in C^{1}(\mathbb{R})$ with $\varphi(t) \equiv 1$ for $t \leq 1-\varepsilon$, $\varphi(t) \equiv 0$ for $t \geq 1$ and $\varphi^{\prime}(t) \leq 0$ for all $t$. Then we define $\gamma(t)=\varphi(t / \rho)$ and choose the test vector $X$ in $(1.5)$ to be

$$
X(z)=-\gamma(d(f(z), a)) J\left(\exp _{f(z)}^{-1} a\right) \text { for } z \in D_{r}\left(z_{0}\right)
$$

and $X(z)=0$ for $z \in D_{R_{1}}(w) \backslash D_{r}\left(z_{0}\right)$. Clearly, $X$ is a vector field along $f$. Because $\gamma(d(f(z), a)) \equiv 0$ on $\partial D_{r}\left(z_{0}\right), X$ belongs to the Sobolev space $H^{1,2}$. We have

$$
\begin{aligned}
\frac{\partial X}{\partial u}= & -\gamma(d(f, a)) J\left(\frac{D}{\partial u} \exp _{f}^{-1} a\right) \\
& +\gamma^{\prime}(d(f, a))\left\langle\exp _{f}^{-1} a, \frac{\partial f}{\partial u}\right\rangle J\left(\exp _{f}^{-1} a\right)(d(f, a))^{-1} \\
& -\gamma(d(f, a)) \frac{\partial J}{\partial u}\left(\exp _{f}^{-1} a\right)
\end{aligned}
$$

A similar formula holds for $\partial X / \partial v$. 
To estimate $(D / \partial u) \exp _{f}^{-1} a$ and $(D / \partial v) \exp _{f}^{-1} a$ we observe that for a $C^{1}$ curve $x(t)$ in the geodesic ball $B_{\rho}(a),(D / \partial t) \exp _{x(t)}^{-1} a$ can be related to a Jacobi field. Namely, if we put $C(s, t)=\exp _{x(t)}\left(s \exp _{x(t)}^{-1} a\right)$, then for each fixed $t$ we obtain a Jacobi field $J_{t}(s)=\partial C(s, t) / \partial t$ along the geodesic $C(\cdot, t)$ with $J_{t}(0)=d x / d t$ and $J_{t}(1)=0 \in T_{a} M$. An easy computation shows $(D / \partial t) \exp _{x(t)}^{-1} a=D J_{t}(0) / \partial s$. This argument can readily be extended to curves of the form $f(z(t))$ for any Sobolev map $f$ and we derive from standard Jacobi field estimates (see, e.g., [Jo1])

$$
\begin{aligned}
& \left\|\frac{D}{\partial u} \exp _{f}^{-1} a+\frac{\partial f}{\partial u}\right\| \leq \frac{\wedge^{2}}{2} d^{2}(f, a)\left\|\frac{\partial f}{\partial u}\right\|, \\
& \left\|\frac{D}{\partial v} \exp _{f}^{-1} a+\frac{\partial f}{\partial v}\right\| \leq \frac{\wedge^{2}}{2} d^{2}(f, a)\left\|\frac{\partial f}{\partial v}\right\| .
\end{aligned}
$$

At this stage we insert a lemma

Lemma 1.1. Weakly holomorphic curves are conformal almost everywhere with respect to a hermitian metric on the target and a conformal metric on the domain. Proof of the lemma. Consider the above situation. We have

$$
\left\langle\frac{\partial f}{\partial u}, \frac{\partial f}{\partial v}\right\rangle=\left\langle\frac{\partial f}{\partial u}, J \frac{\partial f}{\partial u}\right\rangle=-\left\langle J \frac{\partial f}{\partial u}, \frac{\partial f}{\partial u}\right\rangle=-\left\langle\frac{\partial f}{\partial v}, \frac{\partial f}{\partial u}\right\rangle,
$$

hence it is zero. Moreover,

$$
\left\langle\frac{\partial f}{\partial v}, \frac{\partial f}{\partial v}\right\rangle=\left\langle J \frac{\partial f}{\partial u}, J \frac{\partial f}{\partial u}\right\rangle=\left\langle\frac{\partial f}{\partial u}, \frac{\partial f}{\partial u}\right\rangle .
$$

Now we continue the proof of Theorem 1.1. Noting $\left\|\exp _{f}^{-1} a\right\|=d(f, a)$ we deduce from conformality

$$
\left\langle\exp _{f}^{-1} a, \frac{\partial f}{\partial u}\right\rangle^{2}+\left\langle\exp _{f}^{-1} a, \frac{\partial f}{\partial v}\right\rangle^{2} \leq \frac{1}{2} d^{2}(f, a)\|d f\|^{2} .
$$

We also have

$$
\begin{aligned}
& \left\|\frac{\partial J}{\partial u}\left(\exp _{f}^{-1} a\right)\right\| \leq\|D J\|\left\|\frac{\partial f}{\partial u}\right\| d(f, a), \\
& \left\|\frac{\partial J}{\partial v}\left(\exp _{f}^{-1} a\right)\right\| \leq\|D J\|\left\|\frac{\partial f}{\partial v}\right\| d(f, a) .
\end{aligned}
$$

To proceed, we put

$$
I(\rho)=\int_{D_{r}\left(z_{0}\right)}\|d f\|^{2} \varphi\left(\frac{d(f, a)}{\rho}\right) .
$$

$\left(\|d f\|\right.$ is defined in terms of $g$ and $d u^{2}+d v^{2}$.) Since $t \gamma^{\prime}(t)=-\rho(d / d \rho) \varphi(t / \rho)$, there holds

$$
I^{\prime}(\rho)=-\frac{1}{\rho} \int_{D_{r}\left(z_{0}\right)}\|d f\|^{2} d(f, a) \gamma^{\prime}(d(f, a))
$$


Now

$$
\begin{aligned}
J\left(\frac{\partial f}{\partial u}\right) \cdot \frac{\partial X}{\partial u} & +J\left(\frac{\partial f}{\partial v}\right) \cdot \frac{\partial X}{\partial v} \\
= & \gamma\left(\left\langle\frac{\partial f}{\partial u},-\frac{D}{\partial u} \exp _{f}^{-1} a\right\rangle+\left\langle\frac{\partial f}{\partial v},-\frac{D}{\partial v} \exp _{f}^{-1} a\right\rangle\right) \\
& +\gamma^{\prime}\left(\left\langle\frac{\partial f}{\partial u}, \exp _{f}^{-1} a\right\rangle^{2}+\left\langle\frac{\partial f}{\partial v}, \exp _{f}^{-1} a\right\rangle\right) \frac{1}{d(x, a)} \\
& -\gamma\left(\left\langle\frac{\partial f}{\partial u}, \frac{\partial J}{\partial u}\left(\exp _{f}^{-1} a\right)\right\rangle+\left\langle\frac{\partial f}{\partial v}, \frac{\partial J}{\partial v}\left(\exp _{f}^{-1} a\right)\right\rangle\right) .
\end{aligned}
$$

Combining equation (1.5) with (1.9)-(1.12) we then derive

$$
2 I(\rho)-\rho I^{\prime}(\rho) \leq C \rho I(\rho),
$$

where $C$ depends only on $\wedge$ and sup $\|D J\|$. With $\sigma(\rho)=(1+C \rho) e^{2 C \rho}$ this implies by integration for $0<\rho_{1} \leq \rho_{2} \leq \rho^{\prime}$

$$
\frac{\sigma\left(\rho_{1}\right)}{\rho_{1}^{2}} I\left(\rho_{1}\right) \leq \frac{\sigma\left(\rho_{2}\right)}{\rho_{2}^{2}} I\left(\rho_{2}\right) \text {. }
$$

Now we need another lemma.

Lemma 1.2. Let $\psi:(S, h) \rightarrow(M, g)$ be an almost everywhere conformal Sobolev map, with $(M, g)$ isometrically embedded in some $\mathbb{R}^{m}$. Assume that $\psi$ is approximately differentiable at some $z_{0} \in \stackrel{\circ}{S}$, its approximate differential at $z_{0}$ is nonzero, and $z_{0}$ is a Lebesgue point for both $\psi$ and $d \psi$. (We say that $z_{0}$ is a regular point of $\psi$.) Then

$$
\lim _{\rho \rightarrow 0} \sup \frac{1}{\rho^{2}} \int_{\left\{d\left(\psi(z), \psi\left(z_{0}\right)\right)<\rho\right\}}\|d \psi\|^{2} \geq 2 \pi .
$$

(Here the metric $h$ is used to define the norm and the integral. Of course, the Dirichlet energy $\int\|d \psi\|^{2}$ depends only on the conformal class of metrics.) $\psi$ can be replaced by $\left.\psi\right|_{U}$ for any neighborhood $U$ of $z_{0}$.

For a proof we refer to [Grü]. Recall that $\psi$ is said to be approximately differentiable at $z_{0}$ with approximate differential $\nabla \psi\left(z_{0}\right)$ if the following holds: there exists an $x_{0} \in \mathbb{R}^{m}$ such that for every $\varepsilon>0$,

$$
\theta^{2}\left(\mathscr{L}^{2} L \Omega_{\varepsilon}, z_{0}\right)=0 \text {, }
$$

where for a measure $\mu, \theta^{2}(\mu, z):=\lim _{\rho \rightarrow 0} \mu\left(D_{\rho}(z)\right) / \pi \rho^{2}$ whenever the limit exists, $\mathscr{L}^{2}$ denotes the Lebesgue measure induced by $h$, and $\Omega_{\varepsilon}=\{z \in S: z=$ $\left.\exp _{z_{0}}(v),\|v\|<\operatorname{inj}\left(z_{0}\right),\left\|\psi(z)-x_{0}-\nabla \psi\left(w_{0}\right)(v)\right\| \geq \varepsilon\|v\|\right\}$. It is a result of $\mathrm{H}$. Federer that a Sobolev map is approximately differentiable a.e. and its approximate differential coincides with its weak differential a.e.; see [Fe].

Now we continue once again the proof of the theorem. We assume that $z_{0}$ is a regular point of $f$. Letting $\varphi$ approach the characteristic function of $(-\infty, 1)$, letting $\rho_{1}$ tend to zero and $\rho_{2}$ tend to $\rho^{\prime}$, we deduce from $(1.13)$ and Lemma 1.2

$$
\left(\rho^{\prime}\right)^{2} \leq \frac{\sigma\left(\rho^{\prime}\right)}{2 \pi} \int_{D_{r}\left(z_{0}\right)}\|d f\|^{2}, \quad \text { or } \quad \rho^{\prime} \leq C_{1}\left(\int_{D_{r}\left(z_{0}\right)}\|d f\|^{2}\right)^{1 / 2}
$$


for some $C_{1}=C_{1}(C)$. Hence

$$
d(r) \leq C_{1}\left(\int_{D_{r}\left(z_{0}\right)}\|d f\|^{2}\right)^{1 / 2},
$$

because $\rho^{\prime} \in(0, d(r))$ is arbitrary. On the other hand, if $r \leq \frac{1}{2} d\left(z_{0}, \partial D_{R_{1}}(w)\right)$ we can employ Fubini's theorem and the intermediate value theorem in a standard way to find some $\bar{r} \in[r, 2 r]$ such that the trace of $f$ on $\partial D_{\bar{r}}\left(z_{0}\right)$ is continuous and that

$$
d\left(f(z), f\left(z^{\prime}\right)\right) \leq \frac{2 \pi}{\sqrt{\log 2}}\left(\int_{D_{2 r}\left(z_{0}\right)}\|d f\|^{2}\right)^{1 / 2}
$$

for all $z, z^{\prime} \in \partial D_{r}\left(z_{0}\right)$. We apply (1.14) with $r$ replaced by $\bar{r}$, combine it with (1.15) and arrive at

$$
d\left(f\left(z_{1}\right), f\left(z_{1}^{\prime}\right)\right) \leq C_{2}\left(\int_{D_{2 r}\left(z_{0}\right)}\|d f\|^{2}\right)^{1 / 2}
$$

for all Lebesgue points $z_{1}, z_{1}^{\prime} \in D_{r}\left(z_{0}\right)$, where $C_{2}=C_{2}(C)$. By compactness of $D_{R_{1}}(w), \int_{D_{r}(z)}\|d f\|^{2}$ tends to zero uniformly on $D_{R_{1} / 2}(w)$ as $r \rightarrow 0$. Hence (1.16) implies uniform continuity of $f$ on the closure of the set of its regular points in $D_{R_{1} / 2}(w)$. On each component $U$ of the complement of this set the differential of $f$ vanishes and hence $f$ is a constant. Taking limit we see that (1.16) holds for $z_{0} \in \partial U$ and hence $f$ is also continuous on $\partial U$, therewith the everywhere continuity of $f$ is proven.

Intermediate Remark. One easily shows that (1.16) extends to all $z_{0} \in D_{R_{1} / 2}(w)$ and $r \leq R_{1} / 2$, possibly with a larger $C_{2}$.

Once continuity is proven, Hölder continuity and $C^{1}$ regularity follow from known results [Hi, Gi]. Following [Gi and $\mathrm{Ye} 1]$, we present here a variational proof for the purpose of deriving clear estimates and also for the convenience of the reader. The arguments will be needed for treating holomorphic curves with boundary as well. We first note that (1.5) can be rewritten as

$$
\begin{gathered}
\int_{D_{R_{1}}(w)} \frac{\partial f}{\partial u} \cdot \frac{\partial X}{\partial u}+\frac{\partial f}{\partial v} \cdot \frac{\partial X}{\partial v}+J\left(\frac{\partial f}{\partial u}\right) \cdot \nabla J\left(\frac{\partial f}{\partial u}, X\right) \\
+J\left(\frac{\partial f}{\partial v}\right) \cdot \nabla J\left(\frac{\partial f}{\partial v}, X\right)=0
\end{gathered}
$$

We assume $\int_{D_{R_{1}}(w)}\|d f\|^{2}<\left(\rho_{0} / C_{2}\right)^{2}$, which by the above remark implies $f\left(D_{3 R_{1} / 4}(w)\right) \subset B_{\rho_{0}}(f(w))$. We introduce Riemannian normal coordinates on $B_{\rho_{0}}(f(w))$ and conclude from (1.17) that in these coordinates $f$ satisfies the equation

$$
\int_{D_{R_{1}}(w)} \nabla f \cdot \nabla \psi=\int_{D_{R_{1}}(w)} A(f, \nabla f, \nabla f) \cdot \psi
$$

for every $H^{1,2}$ function $\psi$ supported in $\stackrel{\circ}{D}_{R_{1}}(w)$, where $A$ is linear in the second and third arguments and

$$
\sup _{y \neq 0} A(\cdot, y, y)\|y\|^{-2} \leq C_{3}=C_{3}(i(M), \wedge, \sup \|D J\|) .
$$


Note that the metric coefficients $g_{i j}$ involved in, e.g., $(\partial f / \partial u) \cdot(\partial X / \partial u)$ have been eliminated by plugging in $g^{i j}$.

For $z \in D_{R_{1} / 2}(w)$ and $r \leq R_{1} / 4$ we choose a $C^{1}$ function supported in $\stackrel{\circ}{D}_{r}(z)$ with $0<\eta \leq 1, \eta \equiv 1$ on $D_{r / 2}(z)$ and $\|\nabla \eta\| \leq 4 / r$. From (1.18) we obtain

$$
\int_{D_{r}(z)} \nabla f \cdot \nabla\left(\eta^{2}\left(f-(f)_{r}\right)\right)=\int_{D_{r}(z)} A(f, \nabla f, \nabla f) \cdot \eta^{2}\left(f-(f)_{r}\right),
$$

where $(f)_{r}$ is the average of $f$ on $D_{r}(z)$. Easy calculations then yield

$$
\int_{D_{r}\left(z_{0}\right)}\|\nabla f\|^{2} \eta^{2} \leq \frac{C_{4}}{r^{2}} \int_{D_{r}(z) \backslash D_{r / 2}(z)}\left\|f-(f)_{r}\right\|^{2}+C_{4} \int_{D_{r}(z)}\|\nabla f\|^{2} \eta^{2}\left\|f-(f)_{r}\right\| .
$$

The first term on the right-hand side can be handled by the Poincare inequality, while the second one can be absorbed by the left-hand side, provided that $\int_{D_{R_{1}}(w)}\|d f\|^{2}$ is small enough, which we will assume. We arrive at

$$
\int_{D_{r / 2}(z)}\|\nabla f\|^{2} \leq C_{5} \int_{D_{r}(z) \backslash D_{r / 2}(z)}\|\nabla f\|^{2}
$$

Filling the "hole", i.e., adding $C_{5} \int_{D_{r / 2}(z)}\|\nabla f\|^{2}$ to both sides, we see

$$
\int_{D_{r / 2}(z)}\|\nabla f\|^{2} \leq \frac{C_{5}}{1+C_{5}} \int_{D_{r}(z)}\|\nabla f\|^{2},
$$

which implies by a standard iteration argument

$$
\int_{D \rho(z)}\|\nabla f\|^{2} \leq C_{6}\left(\frac{\rho}{r}\right)^{2 \alpha} \int_{D_{r}(z)}\|\nabla f\|^{2}
$$

for $0<\rho \leq r$ and some $\alpha \in(0,1)$ depending only on $C_{5}$. Morrey's Dirichlet growth theorem then yields

$$
\left\|f(z)-f\left(z^{\prime}\right)\right\|^{2} \leq C_{7}\left|z-z^{\prime}\right|^{2 \alpha} \frac{1}{R_{1}^{2 \alpha}} \int_{D_{R_{1}}(w)}\|\nabla f\|^{2}
$$

for $z, z^{\prime} \in D_{R_{1} / 2}(w)$. By rescaling, we may assume $R_{1}=1$. We shall deal with the rescaled $f$, but retain the notations $f$ and $R_{1}$.

Next consider $z \in D_{R_{1} / 4}(w)$ and $r \leq R_{1} / 4$. Let $f^{*}$ be the unique solution of the problem

$$
\Delta f^{*}=0 \text { in } \stackrel{\circ}{D}_{r}(z),\left.\quad f^{*}\right|_{\partial D_{r}(z)}=\left.f\right|_{\partial D_{r}(z)} .
$$

By linear theory we have for $\rho \leq r$ (see [Gi] for the simple argument)

$$
\int_{D_{\rho}(z)}\left\|\nabla f^{*}-\left(\nabla f^{*}\right)_{\rho}\right\|^{2} \leq C_{8}\left(\frac{\rho}{r}\right)^{4} \int_{D_{r}(z)}\left\|\nabla f^{*}-\left(\nabla f^{*}\right)_{r}\right\|^{2} .
$$

Noticing $\int_{D_{r}(z)}\left\|\nabla f^{*}-\left(\nabla f^{*}\right)_{r}\right\|^{2} \leq \int_{D_{r}(z)}\left\|\nabla f-(\nabla f)_{r}\right\|^{2}$, which follows from the minimality of $f^{*}$ and the simple fact $\int_{D_{r}(z)}\left\|\nabla f^{*}-\left(\nabla f^{*}\right)_{r}\right\|^{2}=$ $\inf _{x} \int_{D_{r}(z)}\left\|\nabla f^{*}-x\right\|^{2}$, we derive from (1.23)

$$
\begin{aligned}
\int_{D_{\rho}(z)}\left\|\nabla f-(\nabla f)_{\rho}\right\|^{2} \leq & C_{8}\left(\frac{\rho}{r}\right)^{4} \int_{D_{r}(z)}\left\|\nabla f-(\nabla f)_{r}\right\|^{2} \\
& +C_{9} \int_{D_{r}(z)}\left\|\nabla f-\nabla f^{*}\right\|^{2} .
\end{aligned}
$$


Next we combine the two equations (1.18) and (1.22) and apply (1.21) to deduce

$$
\int_{D_{r}(z)}\left\|\nabla f-\nabla f^{*}\right\|^{2} \leq C_{10} r^{\alpha} \int_{D_{r}(z)}\|\nabla f\|^{2},
$$

whence (1.24) implies

$$
\begin{aligned}
\int_{D_{\rho}(z)}\left\|\nabla f-(\nabla f)_{\rho}\right\|^{2} \leq & C_{11}\left(\frac{\rho}{r}\right)^{4} \int_{D_{r}(z)}\left\|\nabla f-(\nabla f)_{r}\right\|^{2} \\
& +C_{11} r^{\alpha} \int_{D_{r}(z)}\|\nabla f\|^{2} .
\end{aligned}
$$

Now we insert the estimate (1.20) into (1.25) and apply an iteration argument (Lemma 2.1, Chapter 3 in [Gi]) to infer

$$
\int_{D_{\rho}(z)}\left\|\nabla f-(\nabla f)_{\rho}\right\|^{2} \leq C_{12} \rho^{3 \alpha} \int_{D_{R_{1}}(w)}\|\nabla f\|^{2}
$$

Because $z$ is arbitrary, this means $\nabla f \in \mathscr{L}^{2,3 \alpha}\left(D_{R_{1} / 4}(w)\right)$, where $\mathscr{L}^{\rho, \lambda}$ denotes Campanato spaces [Gi]. If $3 \alpha<2$, then $\nabla f \in L^{2,3 \alpha}\left(D_{R_{1} / 4}(w)\right)$, where $L^{\rho, \lambda}$ denotes Morrey spaces. Thus

$$
\int_{D_{\rho}(z)}\|\nabla f\|^{2} \leq C_{13} \rho^{3 \alpha} \int_{D_{R_{1}}(w)}\|\nabla f\|^{2} .
$$

Repeating the above argument with (1.20) replaced by (1.27) we get $\nabla f \in$ $\mathscr{L}^{2,4 \alpha}$. Iterating then yields $\nabla f \in \mathscr{L}^{2, k \alpha}$ for a $k$ such that $k \alpha>2$ (we may assume $\alpha$ to be irrational). Then we obtain from the properties of Campanato spaces [Gi]

$$
\left\|\nabla f(z)-\nabla f\left(z^{\prime}\right)\right\|^{2} \leq C_{14}\left|z-z^{\prime}\right|^{\beta} \int_{D_{R_{1}}(w)}\|\nabla f\|^{2}
$$

for $z, z^{\prime} \in D_{R_{1} / 4}(w)$, where $\beta=k \alpha-2$. It follows that

$$
\|\nabla f(w)\|^{2} \leq \frac{C_{15}}{R_{1}^{2}} \int_{D_{R_{1}}(w)}\|\nabla f\|^{2} .
$$

This estimate holds for the unscaled $f$ by scaling back. It then translates into the estimate (1.1) by standard estimates for normal coordinates on $B_{p_{0}}(f(w))$, the special choice of conformal coordinates and the conformality of $f$. Higher order regularity follows from linear theory.

Remark. Assuming smoothness, the estimate (1.1) can also be derived by a Bochner formula for the energy density $\|d f\|^{2}$, but with $C$ depending on $\left\|D^{2} J\right\|$ also. Besides, such an argument is not applicable to holomorphic curves with boundary.

\section{BOUNDARY REGULARITY OF HOLOMORPHIC CURVES}

We continue to consider the $(M, J, g)$ in the previous section. Let $N$ be a compact totally real submanifold of $M$ and $\widetilde{N}$ a closed submanifold containing $N$.

We construct another metric on $M$. Let $e_{1}, \ldots, e_{n}$ be a local orthonormal frame field on $N$. Because $N$ is totally real, $\left\{e_{1}, \ldots, e_{n}, J e_{1}, \ldots, J e_{n}\right\}$ is a 
frame field. We declare it to be orthonormal. By partition of unity we obtain this way a metric $\tilde{g}$ on $\left.T M\right|_{N}$, which we extend to a tubular neighborhood of $\tilde{N}$. Then we set $\bar{g}=\tilde{g}(J, J)+\tilde{g}$. Interpolating $\bar{g}$ with $g$ we finally arrive at a hermitian metric $g_{0}$ on $M$. We shall use $g_{0}$ throughout the remaining part of this section. In particular, $D$ will denote the Levi-Civita connection of $g_{0}$. A crucial property of $g_{0}$ is that $N$ is pseudo-Lagrangian in $\left(M, J, g_{0}\right)$, i.e., $J\left(T_{p} N\right) \perp T_{p} N$ for every $p \in N$. This follows from the construction of $g_{0}$. The geometric consequence of this fact is the following

Lemma 2.1. (1) Let $f:(S, j) \rightarrow(M, J)$ be a weakly holomorphic curve. If $f$ is $C^{1}$ in a neighborhood of some $z \in \partial S$ and $f(U \cap \partial S) \subset N$ for a neighborhood $U$ of $z$, then $d f(\nu) \perp T_{f(z)} N$, where $\nu$ denotes the inward unit normal of $\partial S$ at $z$.

(2) If $f$ is smooth on $S \backslash\{z\}$ for some $z \in \partial S$ and $f(\partial S \backslash\{z\}) \subset N$, then there holds

$$
\int_{S}\left\langle J \circ d f, D_{J} X\right\rangle=0
$$

(the left-hand side is defined in terms of a Poincare metric on $S$ ) or in local conformal coordinates

$$
\int J\left(\frac{\partial f}{\partial u}\right) \cdot \frac{\partial X}{\partial u}+J\left(\frac{\partial f}{\partial v}\right) \cdot \frac{\partial X}{\partial v}=0
$$

where $X$ is an arbitrary $H^{1,2}$ vector field along $f$ such that $\left.X\right|_{\partial S}$ is tangent to $N$ a.e.

Proof. (1) Let $v \in T_{f(z)} N$. Then $\langle d f(\nu), v\rangle=\langle J(d f(\nu)), J(v)\rangle=\langle d f(j(\nu))$, $J(v)\rangle=0$, because $j(\nu)$ is tangent to $\partial S$.

(2) Delete a neighborhood of $z$ from $S$ and argue in the same fashion as in the proof of (1.5). Boundary integrals arising from integration by parts vanish because of 1$)$.

Definition 2.1. A weakly holomorphic curve with boundary on $N$ is a weakly holomorphic curve $f:(S, j) \rightarrow(M, J)$ such that $f(\partial S) \subset N$ and (2.1) (or (2.2)) holds for all $H^{1,2}$ vector fields $X$ along $f$ whose boundary values are tangent to $N$ a.e. (This definition depends on the choice of a hermitian metric which makes $N$ pseudo-Lagrangian. Note that this definition makes sense only for such a metric.)

Theorem 2.1. Weakly holomorphic curves with boundary on $N$ are smooth everywhere. Moreover, for some positive constants $\delta$ and $C$ depending only on $J, g_{0}$, and $N$ the following holds. Let $(S, j)$ be a compact Riemann surface together with a Poincare metric $h$ such that $\partial S$ consist of geodesics (such a metric always exists) and let $f:(S, j) \rightarrow(M, J)$ be a weakly holomorphic curve with boundary on $N$. Consider $w \in S$ and $0<R \leq \min \{1, \operatorname{inj}(w)\}$, where $\operatorname{inj}(w)$ means the injectivity radius as measured on the double of $S$. If $A(R)=\operatorname{area}\left(\left.f\right|_{B_{R}(w)}\right)<\delta$, then

$$
\sup _{B_{R / 2}(w)}\|d f\|^{2} \leq C A(R) / R^{2} .
$$

Proof. The arguments given in [Jo1, Theorem 2.3.2] (see also [Jo2, Jo3] and [Grü-Hil-Ni]) for proving continuity of minimal surfaces with free boundary 
can easily be adapted to the present situation. The necessary modifications have already been demonstrated in the proof of Theorem 1.1 above, hence we leave the details to the reader. Thus we obtain continuity of $f$ at boundary as well as an estimate for oscillation similar to (1.16). The Hölder continuity and $C^{1}$ regularity arguments in the proof of Theorem 1.1 apply with the following modifications. In suitable conformal coordinates, we are now considering half disks $D_{r}^{+}(z)=\left\{z^{\prime} \in \mathbb{C}:\left|z^{\prime}-z\right| \leq r, \operatorname{Im} z^{\prime} \geq 0\right\}$ with $\operatorname{Im} z=0$. On the other hand, we choose suitable local coordinates on $M$ which map $\widetilde{N}$ into the coordinate plane $\Sigma=\left\{x^{n+1}=\cdots=x^{2 n}=0\right\}$. Moreover, the metric coefficient matrix decomposes as $\left(\begin{array}{c}* 0 \\ 0\end{array}\right)$ along $\Sigma$. Then we have $(A$ is similar to the $A$ in (1.18))

$$
\int_{D_{r}^{+}(z)} \nabla f \cdot \nabla \psi=\int_{D_{r}^{+}(z)} A(f, \nabla f, \nabla f) \cdot \psi
$$

for $\psi \in H^{1,2}\left(D_{r}^{+}(z), \mathbb{R}^{2 n}\right)$ satisfying $\left.\psi\right|_{\partial D_{r}^{+} \backslash I_{r}}=0$ and $\psi\left(I_{r}\right) \subset \Sigma$, where $I_{r}=\partial D_{r}^{+} \cap \mathbb{R}$. Now the change in (1.19) is to replace the average $(f)_{r}$ by $[f]_{r}=$ the average of $f$ on $I_{r}$. Applying the Poincare type inequality in [Yel] and arguing as before we then arrive at

$$
\int_{D_{\rho}^{+}(z)}\|\nabla f\|^{2} \leq C\left(\frac{\rho}{r}\right)^{2 \alpha} \int_{D_{r}(z)}\|\nabla f\|^{2}
$$

for $0<\rho \leq r$. This combined with the interior estimate (1.20) imply a Hölder estimate of the type (1.21). The modification of the $C^{1}$ argument is the following. First, we replace everywhere the average ()$_{r}$ by []$_{r}$. Second, the comparison problem (1.22) is replaced by

$$
\triangle f^{*}=0 \quad \text { in } \stackrel{\circ}{D}_{r}^{+}(z),\left.\quad f^{*}\right|_{\partial D_{r}^{+} \backslash I_{r}}=\left.f\right|_{\partial D_{r}^{+} \backslash I_{r}}, \quad f^{*}\left(I_{r}\right) \subset \Sigma .
$$

The rest goes unchanged. We leave the details to the reader. Higher order regularity again follows from linear theory. We call attention to the paper [Ye1], where a similar and more complicated problem is treated.

Remark. In applications, we shall need to consider the more general situation of a weakly holomorphic curve $f:(S, j) \rightarrow(M, J)$ such that $f(L) \subset N$ and (2.2) holds for $X$ with $\left.X\right|_{L}$ being tangent to $N$ a.e. and $\left.X\right|_{\partial S \backslash L}=0$, where $L$ is an open subset of $\partial S$. Since the arguments for Theorem 2.1 are local in nature, it follows that $f$ is smooth on $\stackrel{\circ}{S} \cup L$ and the estimate (2.3) extends appropriately.

\section{LOCAL BEHAVIOR AND ISOPERIMETRIC INEQUALITY}

Let $(M, J)$ be an almost complex manifold of real dimension $2 n$. We first investigate interior local behavior of holomorphic curves in $(M, J)$. For this purpose it suffices to consider a holomorphic disk $f: D \rightarrow(M, J)$, where $D$ denotes the closed unit disk, whose image is contained in a coordinate chart. We may consider $f$ as a holomorphic curve in $\left(\mathbb{R}^{2 n}, J\right)$ where $J$ is induced from $(M, J)$. We arrange that $f(0)=0$ and $J$ at 0 coincides with the multiplication by $i$ on $\mathbb{R}^{2 n} \simeq \mathbb{C}^{n}$. 
Theorem 3.1. Assume that $f$ is nonconstant. Then there are a natural number $m$ and a nonzero vector $\alpha \in \mathbb{C}^{n}$ such that

$$
f(z)=\alpha z^{m}+O\left(|z|^{m+1}\right) .
$$

Proof. Differentiating (1.2) yields the following differential inequality (see also (1.18))

$$
\|\triangle f\| \leq C\|\nabla f\|^{2}
$$

whence the lemma of Hartman-Wintner [Ha-Wi, Jo1] applies. We obtain

$$
f_{z}=(a+b i) z^{m-1}+o\left(|z|^{m-1}\right)
$$

for some $a, b \in \mathbb{R}^{2 n},\|a\|^{2}+\|b\|^{2} \neq 0$.

The holomorphic property of $f$ implies $a=i b$ by a simple calculation, where $a, b$ are considered as vectors in $\mathbb{C}^{n}$. Integrating (3.3) then yields (3.1) (one first obtains $o\left(|z|^{m}\right)$ but can replace it by $O\left(|z|^{m+1}\right.$ ) because $f$ is smooth).

Corollary. Holomorphic curves have only isolated interior branch points ( $z$ is called a branch point of $f$ if $d f(z)$ is not injective).

Next we study boundary local behavior of holomorphic curves. We replace $D$ in the above discussion by $D^{+}=$closed upper-half unit disk and assume that $f$ maps $\partial D^{+} \cap \mathbb{R}$ into a totally real submanifold $N$. We choose a hermitian metric such that $N$ is pseudo-Lagrangian. Then $f$ meets $N$ orthogonally along $\partial D^{+} \cap \mathbb{R}$ according to Lemma 2.1. In suitable coordinates the inequality (3.2) then holds. Moreover, $f$ meets the coordinate plane $\mathbb{R}^{n}$ orthogonally along $\partial D^{+} \cap \mathbb{R}$. We again arrange that $f(0)=0$ and $J$ at 0 coincides with the multiplication by $i$.

Theorem 3.2. Assume that $f$ is nonconstant. Then there are a natural number $m$ and a nonzero vector $\alpha \in \mathbb{C}^{n}$ such that

$$
f(z)=\alpha z^{m}+O\left(|z|^{m+1}\right) .
$$

Proof. We extend $f$ to $D$ by reflection across the coordinate plane $\mathbb{R}^{n}$. Although (3.2) does not hold on $D$, we still have

$$
\left\|\int_{\partial \Omega} \varphi f_{z}\right\| \leq C \int_{\Omega}\left(\left\|\varphi_{\bar{z}}\right\|\left\|f_{z}\right\|+\|\varphi\|\left(\left\|f_{z}\right\|+\|f\|\right)\right)
$$

for every $\Omega \subset D$ with Lipschitz boundary and every $\varphi \in C^{1}(\Omega, \mathbb{C}) \cap \operatorname{Lip}(\bar{\Omega}, \mathbb{C})$. Hence the lemma of Hartman-Wintner is still applicable.

Corollary. Holomorphic curves with boundary on totally real submanifolds have only isolated boundary branch points.

With the above analysis of local behavior it is now easy to derive an isoperimetric inequality for holomorphic curves in $(M, J)$.

For this purpose we assume that $(M, J)$ has bounded geometry and fix a prefered hermitian metric $g$.

Theorem 3.3. There are a universal positive number $C$ and a positive number $\sigma$ depending only on $i(M), \sup |K|$ and sup $\|D J\|$ such that the following holds. Let $f:(S, j) \rightarrow(M, J)$ be a holomorphic curve and $\Omega \subset S$, then

$$
\operatorname{area}\left(\left.f\right|_{\Omega}\right) \leq C \text { length }\left(\left.f\right|_{\partial \Omega}\right)^{2},
$$

provided that area $\left(\left.f\right|_{\Omega}\right) \leq \sigma$. 
Proof. Since $f$ has only isolated branch points, we can assume $f$ to be an immersion by a simple cutting argument (the boundary $\partial S$ can be approximated from interior). By differentiation we obtain from (1.2)

$$
\triangle f+J^{-1}\left(D J\left(\frac{\partial f}{\partial u}, \frac{\partial f}{\partial u}\right)+D J\left(\frac{\partial f}{\partial v}, \frac{\partial f}{\partial v}\right)\right)=0 .
$$

Because $f$ is conformal, this implies $\|H\| \leq C_{1}=C_{1}(\sup \|D J\|)$, where $H$ denotes the mean curvature vector field of $f$. Then it follows from [Ho-Spru] that

$$
C_{2} \operatorname{area}\left(\left.f\right|_{\Omega}\right)^{1 / 2} \leq \operatorname{length}\left(\left.f\right|_{\partial \Omega}\right)+\int_{\Omega}\|H\| d v,
$$

where $C_{2}$ is universal and $d v$ is the induced volume form, provided that area $\left(\left.f\right|_{\Omega}\right) \leq a_{1}=a_{1}(i(M)$, sup $|K|)$. But (3.7) implies $2 C_{2}$ area $\left(\left.f\right|_{\Omega}\right)^{1 / 2} \leq$ length $\left(\left.f\right|_{\partial \Omega}\right)$, if $C_{1}$ area $\left(\left.f\right|_{\Omega}\right)^{1 / 2} \leq C_{2} / 2$.

The isoperimetric inequality (3.5) is not quite enough for dealing with holomorphic curves with boundary. We need the following

Theorem 3.4. Let $N$ be a compact totally real submanifold of $(M, J)$ and $g_{0}$ be constructed as in $\S 2$. There are a tubular neighborhood $U$ of $N$, a universal positive number $\sigma$ depending on $J, g_{0}$ and $N$ such that the following holds. If $f:(S, j) \rightarrow(M, J)$ is a holomorphic curve with $f(S) \subset U$, and $\Omega \subset S$, then

$$
\operatorname{area}\left(\left.f\right|_{\Omega}\right) \leq C \text { length }\left(\left.f\right|_{\partial \Omega \backslash L}\right)^{2},
$$

provided that area $\left(\left.f\right|_{\Omega}\right) \leq \sigma$ and $L$ is an open set of $\partial \Omega$ with $f(L) \subset N$.

Proof. Because of (3.6) and Lemma 2.1 we know that $f$ has bounded mean curvature and meets $N$ orthogonally along $L$. Hence it follows from [Ye3] that

$$
C \text { area }\left(\left.f\right|_{\Omega}\right)^{1 / 2} \leq \text { length }\left(\left.f\right|_{\partial \Omega \backslash L}\right)+\int_{\Omega}\|H\| d v,
$$

which implies the result.

\section{GROMOV'S COMPACTNESS THEOREM}

Let $(M, J)$ be a compact almost complex manifold of real dimension $2 n$ equipped with a hermitian metric $g$. We first prove Theorem $0.1(\mathrm{i})$. Let $f_{k}:\left(S, j_{k}\right) \rightarrow(M, J)$ be a sequence of closed holomorphic curves of a fixed topological type and uniformly bounded area. We equip $S$ with a Poincaré metric $h_{k}$ for $j_{k}$, where $h_{k}$ is normalized to have volume one in case $S$ has genus one. There are two possible cases.

Case 1. $\left\{j_{k}\right\}$ lie in a compact set of the Riemann moduli space of complex structures on $S$.

Then there is a uniform positive lower bound $r_{0}$ for the injectivity radius of $\left(S, h_{k}\right)$ (see, e.g., [Ye2]). Pulling back each $h_{k}$ by a suitable diffeomorphism and passing to a subsequence, we may assume that $\left\{h_{k}\right\}$ converges smoothly [Ye2]. In particular, $C h_{k} \leq h_{k^{\prime}} \leq C^{-1} h_{k}$ for some $C>0$ and all $k, k^{\prime}$. 
For fixed $m \geq 1$ and $k \geq 1$, let $\left\{B_{1 / m}\left(w_{i}\right)\right\}_{i}$ be a maximal family of disjoint geodesic balls on $\left(S, h_{k}\right)$. Then $\left\{B_{2 / m}\left(w_{i}\right)\right\}_{i}$ covers $S$. By BishopGromov volume comparison [Ga], each point $\bar{w} \in S$ is contained in at most $\sup _{w} \operatorname{vol}\left(B_{9 / m}(w)\right) / \operatorname{vol}\left(B_{1 / m}(w)\right) \leq l_{0}$ balls $B_{4 / m}\left(w_{i}\right)$, where $l_{o}$ is universal. It follows that

$$
\sum_{i} \int_{B_{4 / m}\left(w_{i}\right)} e\left(f_{k}\right) \leq l_{0} a
$$

where $e\left(f_{k}\right)=\left\|d f_{k}\right\|^{2}$ is the energy density of $f_{k}$ measured in $h_{k}$, the integration is with respect to the volume form of $h_{k}$, and $a=\sup _{k}$ area $\left(f_{k}\right)$. (Note that energy $=2$ area because of conformality of $f_{k}$ (Lemma 1.1).) Let $B_{1}, B_{2}, \ldots, B_{l}$ be all "bad" balls in $\left\{B_{4 / m}\left(w_{i}\right)\right\}_{i}$, i.e., balls for which

$$
\int_{B_{i}} e\left(f_{k}\right) \geq \frac{\delta}{4}
$$

holds, where $\delta$ is given in Theorem 1.1. Then $l \leq 4 l_{0} a / \delta$. Now fix $m$ but let $k$ vary. By passing to a subsequence of $f_{k}$, we may assume that the center points of the bad balls constitute $l$ converging sequences with limit points $w_{m 1}, \ldots, w_{m l}$, where $l \leq 2 l_{0} a / \delta$. Next we pass to a subsequence of $\{m\}$ such that $\left\{w_{m 1}, \ldots, w_{m l}\right\}$ converge to points $\left\{w_{1}, \ldots, w_{l}\right\}$. We conclude with an easy argument.

Lemma 4.1. For a subsequence of $f_{k}$, still denoted $f_{k}$ (we shall always use the same notation $f_{k}$ later on, when we pass to a subsequence of $f_{k}$ ) the following is true. Let $\Gamma \subset S$ be the set of blow-up points $w$ characterized by the following condition

$$
\lim _{r \rightarrow 0} \lim _{k \rightarrow \infty} \sup \int_{B_{r}(w)} e\left(f_{k}\right) \geq \frac{\delta}{2},
$$

where $B_{r}(w)$ is measured in $h_{k}$. Then $\# \Gamma \leq 4 l_{0} a / \delta$.

This lemma and a simple covering argument imply: for each $r>0$, there are $\varepsilon>0$ and $K \in \mathbb{N}$ such that $\int_{B_{\varepsilon}(w)} e\left(f_{k}\right)<\delta$ whenever $k \geq K$ and $d(w, \Gamma) \geq$ $r$, where geometric quantities are measured in $h_{k}$. For a subsequence of $f_{k}$, Theorem 1.1 then implies uniform gradient bounds on compact subsets of $S \backslash \Gamma$. Higher order estimates follow from standard linear elliptic theory. We arrive at

Lemma 4.2. A subsequence of $\left\{f_{k}\right\}$ converges smoothly on $S \backslash \Gamma$ to some $f_{\infty}$.

By Theorem 1.1, $f_{\infty}$ extends smoothly across $\Gamma$. This is a part of the soughtafter cusp-curve limit of the sequence $f_{k}$. For convenience, the arguments leading to Lemma 4.1 and Lemma 4.2 will be referred to as Sacks-Uhlenbeck Convergence Scheme. Before proceeding, we notice the following area relation after passing to a subsequence of $\left\{f_{k}\right\}$ :

$$
\lim _{k \rightarrow \infty} \operatorname{area}\left(f_{k}\right)=\operatorname{area}\left(f_{\infty}\right)+\sum_{w \in \Gamma} a(w),
$$

where $\Gamma$ is the set of blow-up points for this new sequence $\left\{f_{k}\right\}$ and the area loss $a(w)$ of $\left\{f_{k}\right\}$ at $w$ is defined to be half of the left-hand side of (4.3). 
Next we recover blow-up limits at blow-up points. Consider $w_{0} \in \Gamma$. We choose conformal coordinates for $h_{k}$ on $B_{\varepsilon_{k}}\left(w_{0}\right)$, where

$$
\varepsilon_{k}=\frac{1}{2} \min \left\{d\left(w_{i}, w_{j}\right): w_{i}, w_{j} \in \Gamma, w_{i} \neq w_{j}\right\}
$$

(distance being measured in $h_{k}$ ).

As in the proof of Theorem 1.1, we choose either stereographic, euclidean or Poincaré coordinates centered at $w_{0}$. Then we can consider $\left.f_{k}\right|_{B_{\varepsilon_{k}}\left(w_{0}\right)}$ as defined on $D_{\bar{\varepsilon}_{k}}=D_{\bar{\varepsilon}_{k}}(0)$, which is a holomorphic curve with respect to the standard complex structure of $\mathbb{C}$, where $\bar{\varepsilon}_{k} \geq c \min \left\{1, r_{0}\right\}$ for a universal positive number $c$. From now on we use the euclidean metric on $\mathbb{C}$. Set $b_{k}=\max \left\{\left\|d f_{k}(w)\right\|: w \in D_{\bar{\varepsilon}_{k}}\left(w_{0}\right)\right\}$ and let $z_{k}$ be a point at which $b_{k}$ is achieved. Because of (4.3), we have $\lim _{k \rightarrow \infty} b_{k}=\infty$. Since $f_{k}$ converge smoothly on $S \backslash \Gamma$, we conclude that $z_{k} \rightarrow 0$ as $k \rightarrow \infty$. Hence we can choose a sequence $r_{k} \rightarrow 0$ and a subsequence of $f_{k}$ such that

$$
\begin{aligned}
& \lim _{k \rightarrow \infty} \operatorname{area}\left(\left.f_{k}\right|_{D_{r_{k}^{2}}\left(z_{k}\right)}\right)=a\left(w_{0}\right), \\
& \lim _{k \rightarrow \infty} \operatorname{area}\left(\left.f_{k}\right|_{D_{r_{k}}\left(z_{k}\right) \backslash D_{r_{k}^{2}}\left(z_{k}\right)}\right)=0,
\end{aligned}
$$

as well as

$$
R_{k}^{\prime}:=b_{k} r_{k}^{2} \rightarrow \infty \text { as } k \rightarrow \infty
$$

We set $R_{k}=b_{k} r_{k}$. Translating and dilating by factor $b_{k}$ we may consider $\tilde{f}_{k}=\left.f_{k}\right|_{D_{r_{k}}(z)}$ as defined on $D_{R_{k}}$. Then we also have

$$
\sup \left\|d \tilde{f}_{k}\right\|=\left\|d \tilde{f}_{k}\right\|(0)=1 \text {. }
$$

From linear elliptic theory we deduce that (a subsequence of) $\left\{\tilde{f}_{k}\right\}$ converges locally smoothly to a holomorphic curve $\tilde{f}_{\infty}: \mathbb{C} \rightarrow(M, J)$. It also follows from (4.8) that $\left\|\tilde{f}_{\infty}\right\|(0)=1$, whence $\tilde{f}_{\infty}$ is nonconstant. Composing $\tilde{f}_{\infty}$ with the stereographic projection we get a nonconstant holomorphic sphere with an isolated singularity, which can be removed on account of Theorem 1.1. We shall refer to the above procedure of handling $w_{0}$ as Procedure 1.

We define the area loss of $\left\{\tilde{f}_{k}\right\}$ at $\infty$ to be

$$
a_{\infty}=\lim _{R \rightarrow \infty} \limsup _{k \rightarrow \infty} \operatorname{area}\left(\left.\tilde{f}_{k}\right|_{D_{R_{k}} \backslash D_{R}}\right) .
$$

Then

$$
a\left(w_{0}\right)=\operatorname{area}\left(\tilde{f}_{\infty}\right)+a_{\infty}
$$

We also have

$$
\lim _{k \rightarrow \infty} \operatorname{area}\left(\left.\tilde{f}_{k}\right|_{D_{R_{k}} \backslash D_{R_{k}^{\prime}}}\right)=0
$$

which follows from (4.6). The sequence of pairs $\left\{R_{k}, R_{k}^{\prime}\right\}$ satisfies both (4.11) and the relations $R_{k}^{\prime} \rightarrow \infty, R_{k} / R_{k}^{\prime} \rightarrow \infty$. Such a sequence will be called a tail for $\left\{\tilde{f}_{k}\right\}$. Next we choose a sequence $T_{k} \rightarrow \infty$ and a subsequence of $\left\{\tilde{f}_{k}\right\}$ such that $T_{k}^{2}<R_{k}^{\prime}$ and

$$
a_{\infty}=\lim _{k \rightarrow \infty} \operatorname{area}\left(\left.\tilde{f}_{k}\right|_{D_{R_{k}^{\prime}} \backslash D_{T_{k}^{2}}}\right)
$$




$$
\lim _{k \rightarrow \infty} \operatorname{area}\left(\left.\tilde{f}_{k}\right|_{D_{T_{k}^{2}} \backslash D_{T_{k}}}\right)=0 .
$$

We then define the neck limit of $\left\{\tilde{f}_{k}\right\}$ to be

$$
l_{\infty}=\limsup _{R \rightarrow \infty} \sup _{T_{k}^{2} \leq R \leq R_{k}^{\prime}} \operatorname{length}\left(\left.\tilde{f}_{k}\right|_{\partial D_{R}}\right) .
$$

We assume that the area loss $a_{\infty}$ is nonzero, otherwise the area loss $a\left(w_{0}\right)$ is completely captured by $\tilde{f}_{\infty}$ and we are done with the blow-up point $w_{0}$.

Then we claim $l_{\infty}>0$. Suppose the contrary. Let $\sigma$ be the number given in Theorem 3.3. For each $R$ and $k$ with $T_{k}^{2} \leq R \leq R_{k}^{\prime}$, let $R=R^{(0)}<R^{(1)}<$ $\cdots<R^{(m)}=R_{k}^{\prime}$ be chosen such that area $\left(\left.\tilde{f}_{k}\right|_{D_{R^{(i+1)}} \backslash D_{R^{(i)}}}\right) \leq \sigma$ for all $i$ and $m \leq a / \sigma$. Then it follows from Theorem 3.3 that

$$
\operatorname{area}\left(\left.\tilde{f}_{k}\right|_{D_{R^{(i+1)}} \backslash D_{R^{(i)}}}\right) \leq c\left(\text { length }\left(\left.\tilde{f}_{k}\right|_{\partial D_{R^{(i+1)}}}\right)+\operatorname{length}\left(\left.\tilde{f}_{k}\right|_{\partial D_{R^{(i)}}}\right)\right)^{2} .
$$

Summing up we deduce from the supposition $l_{\infty}=0$ and (4.7) that $a_{\infty}=0$, a contradiction.

To recover the area loss $a_{\infty}$, we rescale to capture another holomorphic sphere.

Choose a subsequence of $f_{k}$ and a sequence $\bar{R}_{k} \rightarrow \infty, T_{k}^{2} \leq \bar{R}_{k} \leq R_{k}^{\prime}$ such that $l_{\infty}=\lim _{k \rightarrow \infty} \operatorname{length}\left(\left.\tilde{f}_{k}\right|_{\partial D_{\bar{R}_{k}}}\right)$. Then we put $f_{k}^{(1)}(z)=\tilde{f}_{k}\left(\bar{R}_{k} z\right)$. Note that the domains of $f_{k}^{(1)}$ approach $\mathbb{C}$ because $\bar{R}_{k} \leq R_{k}^{\prime}$ and $R_{k} / R_{k}^{\prime} \rightarrow \infty$. Applying Sacks-Uhlenbeck Convergence Scheme, we obtain a set $\Gamma^{(1)} \subset \mathbb{C} \backslash\{0\}$ of (finitely many) blow-up points and local smooth convergence of (a subsequence of) $f_{k}^{(1)}$ on $\mathbb{C} \backslash\left(\Gamma^{(1)} \cup\{0\}\right)$. Call the limit $f_{\infty}^{(1)}$. We have the following area relation after passing to a subsequence of $\left\{f_{k}^{(1)}\right\}$ :

$$
a_{\infty}=\operatorname{area}\left(f_{\infty}^{(1)}\right)+\sum_{w \in \Gamma^{(1)}} a^{(1)}(w)+a_{\infty}^{(1)}+\hat{a}(0),
$$

where $a^{(1)}(w)$ is the area loss of $\left\{f_{k}^{(1)}\right\}$ at $w, a_{\infty}^{(1)}$ is the area loss of $\left\{f_{k}^{(1)}\right\}$ at $\infty$, and $\hat{a}(0)$ is the reduced area loss of $\left\{f_{k}^{(1)}\right\}$ at 0 defined as

$$
\hat{a}(0)=\lim _{r \rightarrow 0} \lim _{k \rightarrow \infty} \sup \operatorname{area}\left(\left.f_{k}^{(1)}\right|_{D_{r} \backslash D_{T_{k} / \overline{k_{k}}}}\right) .
$$

Since length $\left(\left.f_{k}^{(1)}\right|_{\partial D_{1}}\right) \rightarrow l_{\infty} \neq 0$, one of the following must happen:

(1) $\Gamma^{(1)}$ is nonempty,

(2) $f_{\infty}^{(1)}$ is nonconstant.

If $f_{\infty}^{(1)}$ is nonconstant, it yields a nonconstant holomorphic sphere. The procedure of handling $a_{\infty}$ so far will be called Procedure 2.

To handle the area loss $a_{\infty}^{(1)}$, we note that if $R_{k}^{\prime} / \bar{R}_{k}$ are uniformly bounded, then (4.1) implies $a_{\infty}^{(1)}=0$. Thus, if $a_{\infty}^{(1)} \neq 0$, then (after passing to a subsequence) the sequence $\left\{R_{k} / \bar{R}_{k}, R_{k}^{\prime} / \bar{R}_{k}\right\}$ is a tail for $\left\{f_{k}^{(1)}\right\}$ and Procedure 2 can be applied to handle $a_{\infty}^{(1)}$. To treat $\hat{a}(0)$, we make an inversion across $\partial D_{1}$ and obtain from $\left\{f_{k}^{(1)}\right\}$ a sequence $\left\{f_{k}^{(2)}\right\}$. Then

$$
\hat{a}(0)=\lim _{R \rightarrow \infty} \limsup _{k \rightarrow \infty} \operatorname{area}\left(\left.f_{k}^{(2)}\right|_{D_{R_{k} / T_{k}} \backslash D_{R}}\right),
$$


thus $\hat{a}(0)$ is the area loss of $\left\{\left.f_{k}^{(2)}\right|_{D_{R_{k}} / T_{k}}\right\}$ at $\infty$. If $\bar{R}_{k} / T_{k}^{2}$ are uniformly bounded, (4.13) implies $\hat{a}(0)=0$. Hence, if $\hat{a}(0) \neq 0$, then (after passing to a subsequence) the sequence $\left\{\bar{R}_{k} / T_{k}, \bar{R}_{k} / T_{k}^{2}\right\}$ is a tail for $\left\{f_{k}^{(2)} \mid D_{R_{k} / T_{k}}\right\}$ and Procedure 2 can again be applied. Now we insert a lemma.

Lemma 4.3. There is a positive number $\sigma$ depending on $(J, g)$ such that area $(f)$ $\geq \sigma$ for each nonconstant closed holomorphic curve $f$.

Proof of Lemma 4.3. Choose $\sigma$ to be the $\sigma$ in Theorem 3.3. Then area $(f)<\sigma$ implies area $(f)=0$ because $f$ is closed.

Now we can apply Procedure 1 and Procedure 2 repeatedly. We can argue by induction on captured holomorphic spheres. Because of Lemma 4.3, after finitely many steps there will be no area loss and the initial area loss $a\left(w_{0}\right)$ is completely captured by holomorphic spheres obtained in the process. The same argument is then applied to every blow-up point of the initial sequence $\left\{f_{k}\right\}$. Let $f_{\infty}^{1}, \ldots, f_{\infty}^{(m)}$ be all holomorphic spheres obtained in the process. Then

$$
\lim _{k \rightarrow \infty} \operatorname{area}\left(f_{k}\right)=\operatorname{area}\left(f_{\infty}\right)+\sum_{i=1}^{m} \operatorname{area}\left(f_{\infty}^{i}\right) .
$$

It is then easy to see from the construction how to put $f_{\infty}, f_{\infty}^{1}, \ldots, f_{\infty}^{(m)}$ together to make a cusp-curve $f$ such that $f_{k}$ converge to $f$ in the $C^{l}$ topology on the space of cusp-curves. (That all $C^{l}$ topologies are equivalent follows from Theorem 1.1 and linear theory. We leave the details to the reader.)

Case 2. $\left\{j_{k}\right\}$ does not lie in a compact set of the Riemann moduli space of complex structures on $S$.

In other words, the conformal (complex) structures $j_{k}$ degenerate. We assume $\operatorname{genus}(S)>1$ and leave the easier case $\operatorname{genus}(S)=1$ to the reader. The following fact is well-known, a detailed proof can be found in [Ye2].

Lemma 4.4. There exist simple closed geodesics $\alpha_{k 1}, \ldots, \alpha_{k m}$ ( $m \leq 3 \operatorname{genus}(S)$ - 3) on $\left(S, h_{k}\right)$, component decompositions $S \backslash \bigcup_{i=1}^{m} \alpha_{k i}=\bigcup_{l=1}^{\bar{m}} S_{k}^{l}$, such that length $\left(\alpha_{k i}\right) \rightarrow 0$ as $k \rightarrow \infty$ and each sequence $\left\{\left(S_{k}^{l}, h_{k}\right)\right\}_{k}$ converges to a connected, noncompact and complete hyperbolic surface $S^{l}$ with finitely many annulus ends and finite area. The convergence is in the following sense: there are embeddings $\Phi_{k l}: S_{k}^{l} \rightarrow S^{l}$ such that $\Phi_{k l}\left(S_{k}^{l}\right)$ approach $S^{l}$ and $\left(\Phi_{k l}^{-1}\right)^{*} h_{k}$ converge locally smoothly to the metric of $S^{l}$.

Then $\left(\Phi_{k l}^{-1}\right)^{*} j_{k}$ also converge locally smoothly to the complex structure $J_{l}$ of $S^{l}$. Conformally, the annulus ends of $\left(S^{l}, J_{l}\right)$ are punctures and the Riemann surfaces $\left(S^{l}, J_{l}\right)$ naturally compactify with a point added to each annulus end, the compactified surfaces are denoted $\left(\bar{S}^{l}, J_{l}\right)$. Moreover, there are annulus regions $A_{k i}$ on $S$ with the following properties:

(1) $\alpha_{k i} \subset A_{k i}$

(2) there are conformal maps $\varphi_{k i}: D_{l_{k i}} \backslash \stackrel{\circ}{D}_{1} \rightarrow\left(A_{k i}, j_{k}\right)$ with $l_{k i}=$ $\exp \left(1 /\right.$ length $\left.\left(\alpha_{k i}\right)\right)$ such that $\varphi_{k i}^{*} h_{k}=\eta_{k i}^{2}(r)\left(d r^{2}+r^{2} d \theta^{2}\right)$ for some $\eta_{k i}$ and for any $b>1$,

$$
\lim _{k \rightarrow \infty} \int_{1}^{b} \eta_{k i}(r) d r=\int_{1}^{b} \frac{d r}{r|c+\log r|}
$$




$$
\lim _{k \rightarrow \infty} \int_{l_{k i} / b}^{l_{k i}} \eta_{k i}(r) d r=\int_{1}^{b} \frac{d r}{r|c-\log r|},
$$

where $c>0$ depends only on genus $(S)$;

(3) If a component $L$ of $\partial A_{k i}$ is contained in some $S_{k}^{l}$, then $\Phi_{k l}(L)$ stays in a compact region of $S^{l}$ independent of $k$.

$A_{k i}$ is called a collar about $\alpha_{k i}$. As $k \rightarrow \infty, A_{k i} \backslash \alpha_{k i}$ yield two annulus ends of some $S^{l}$.

Now using $\Phi_{k l}$ we can consider $f_{k}$ as defined on subregions of $\tilde{S}:=\bigcup_{l} S^{l}$, which approach $\tilde{S}$ as $k \rightarrow \infty$. We choose an exhaustion of $\tilde{S}$ by compact regions and apply Sacks-Uhlenbeck Convergence Scheme on these regions. Then we obtain a set $\Gamma$ of (finitely many) blow-up points, a subsequence of $\left\{f_{k}\right\}$ such that $f_{k}$ converge locally smoothly on $\tilde{S} \backslash \Gamma$ to some $f_{\infty}$. We can consider $f_{\infty}$ as defined on $\bar{S}:=\bigcup_{l} \bar{S}^{l}$, which is smooth on acount of Theorem 1.1. Moreover, we have

$$
\lim _{k \rightarrow \infty} \operatorname{area}\left(f_{k}\right)=\operatorname{area}\left(f_{\infty}\right)+\sum_{w \in \Gamma} a(w)+\sum_{A \in \mathscr{A}} a(A)
$$

where $\mathscr{A}$ is the set of the annulus ends of $\tilde{S}$ and $a(A)$ is the area loss of $\left\{f_{k}\right\}$ at $A$ defined as follows. Choose decreasing regions $A_{l}$ on $A$ such that each $\partial A_{l}$ is a circle and length $\left(\partial A_{l}\right) \rightarrow 0, \operatorname{dist}\left(\partial A_{1}, \partial A_{l}\right) \rightarrow \infty$ as $l \rightarrow \infty$. Then we set

$$
a(A)=\lim _{l \rightarrow \infty} \limsup _{k \rightarrow \infty} \operatorname{area}\left(\left.f_{k}\right|_{A_{l}}\right) .
$$

This definition is independent of the choice of $\left\{A_{l}\right\}$. The area losses $a(w)$ can be treated in the same way as in Case 1. To recover the $a(A)$ 's, we employ the collars described in Lemma 4.4. Consider some $\left\{A_{k i}\right\}_{k}$ which yield two annulus ends $A, A^{\prime}$ of $\tilde{S}$. Let $\tilde{f}_{k}=f_{k} \circ \varphi_{k i}$. The idea is that the area loss of $\left\{\tilde{f}_{k}\right\}$ at $\infty$ should be $a(A)+a\left(A^{\prime}\right)$, but some adjustment is needed. Let $\beta_{k i}, \beta_{k i}^{\prime}$ be the image of $\partial D_{l_{k i}}$ and $\partial D_{1}$ under $\Phi_{k} \circ \varphi_{k i}$, where $\Phi_{k}$ denotes the union of $\left\{\Phi_{k l}\right\}_{l}$. Because of property (3) of $A_{k i}$, we can assume that $\left\{\beta_{k i}\right\}_{k}$ converges to a circle $\beta$ on $A$ and $\left\{\beta_{k i}^{\prime}\right\}_{k}$ converges to a circle $\beta^{\prime}$ on $A^{\prime}$. Let $\tilde{A}, \tilde{A}^{\prime}$ be the regions on $A, A^{\prime}$ with boundary $\beta, \beta^{\prime}$ respectively. From (4.16), (4.17) it is not hard to see

$$
\begin{gathered}
\operatorname{area}\left(\left.f_{\infty}\right|_{A}\right)=\lim _{b \rightarrow \infty} \lim _{k \rightarrow \infty} \operatorname{area}\left(\left.\tilde{f}_{k}\right|_{D_{l_{k i}} \backslash D_{l_{k i} b^{-1}}}\right), \\
\operatorname{area}\left(\left.f_{\infty}\right|_{A^{\prime}}\right)=\lim _{b \rightarrow \infty} \lim _{k \rightarrow \infty} \operatorname{area}\left(\left.\tilde{f}_{k}\right|_{D_{b} \backslash D_{1}}\right) .
\end{gathered}
$$

Now we choose a sequence $b_{k} \rightarrow \infty$ and a subsequence of $\left\{\tilde{f}_{k}\right\}$ such that $l_{k i} b_{k}^{-2} \rightarrow \infty$ and

$$
\begin{gathered}
\operatorname{area}\left(\left.f_{\infty}\right|_{A}\right)=\lim _{k \rightarrow \infty} \operatorname{area}\left(\left.\tilde{f}_{k}\right|_{D_{l_{k i}} \backslash D_{l_{k i} b^{-1}}}\right), \\
\operatorname{area}\left(\left.\tilde{f}_{k}\right|_{\left.D_{l_{k} b_{k}^{-1}} \backslash D_{l_{k i} b_{k}^{-2}}\right)=0 .} .\right.
\end{gathered}
$$


Then one easily sees that the area loss $a_{\infty}$ of $\left\{\left.\tilde{f}_{k}\right|_{D_{l_{k} b_{k}}{ }^{-1}}\right\}$ at $\infty$ equals $a(A)+$ $a\left(A^{\prime}\right)$. Moreover, the sequence $\left\{l_{k i} b_{k}^{-1}, l_{k i} b_{k}^{-2}\right\}$ is a tail for this mapping sequence. Hence the arguments in Case 1 can be applied to recover $a(A)+a\left(A^{\prime}\right)$. Since all annulus ends can be treated this way, all $a(A)$ can be recovered. Let $f_{\infty}^{1}, \cdots, f_{\infty}^{m}$ be all holomorphic spheres obtained in the process. Then

$$
\lim _{k \rightarrow \infty} \operatorname{area}\left(f_{k}\right)=\operatorname{area}\left(f_{\infty}\right)+\sum_{i=1}^{m} \operatorname{area}\left(f_{\infty}^{i}\right) .
$$

It is again easy to see how to put $f_{\infty}, f_{\infty}^{1}, \ldots, f_{\infty}^{m}$ together to make a cuspcurve $f$ such that $f_{k}$ converge to $f$. (The convergence of complex structures involved is easily verified.)

We conclude that in both Case 1 and Case 2, a subsequence of $\left\{f_{k}\right\}$ converges to a cusp-curve. To complete the proof of Theorem 0.1(i), we need to consider the general case that $\left\{f_{k}\right\}$ is a sequence of cusp-curves. But the component holomorphic curves of $\left\{f_{k}\right\}$ can be handled individually, whence we are done.

To prove Theorem 0.1 (ii), we notice that only the case $m=0$ needs to be considered, because conformal automorphism groups of higher genus are compact. With the above arguments for Theorem 0.1(i) available, the proof given by McDuff in [Mc] can be quoted word by word, therewith Theorem 0.1 is proven.

The above proof for Theorem 0.1 carries over to the boundary case Theorem 0.2 with a few modifications. First, instead of Theorem 1.1, Theorem 2.1 has to be used everywhere.

Second, one notices that at a boundary blow-up point, nonconstant holomorphic curves defined on $\bar{H}^{2}$, the closed half-space, are obtained as rescaling limits. Since $\bar{H}^{2}$ is conformally equivalent to the unit disk, they can be converted into nonconstant holomorphic disks.

Third, besides the lower area bound for nonconstant holomorphic curves provided by Lemma 4.3 , we also need the following analogue for holomorphic disks.

Lemma 4.5. Let $N$ be a compact, totally real submanifold of $(M, J)$. Then there is a positive number $\varepsilon$ depending on $M, N$, and $J$ such that area $(f) \geq$ $\varepsilon$ for every nonconstant holomorphic disk with boundary on $N$.

Proof. One possible argument is to apply Theorem 3.4. But we present another argument. By the gradient estimate in Theorem 2.1, there is an $\varepsilon>0$ such that, if a holomorphic disk $f$ with boundary on $N$ has area less than $\varepsilon$, then its image is contained in a coordinate chart. Moreover, in suitable coordinates we have (cf. §2)

$$
\triangle f+A(f, \nabla f, \nabla f)=0,
$$

where $A$ is similar to the $A$ in (1.18) and $\nu$ denotes the inward unit normal of $\partial D_{1}$. We also have $f(0)=0$. Let $\rho(x)=\|x\|^{2}$. A simple computation using (4.23) shows $\Delta(\rho \circ f) \geq 0$, provided that $\varepsilon$ has been chosen small enough. But 
(4.24) implies $\partial(\rho \circ f) / \partial \nu \equiv 0$ on $\partial D_{1}$, hence we conclude by the maximum principle that $f$ is a constant map.

Remark. By a monotonicity argument, one can show that Lemma 4.5 holds for holomorphic curves of arbitrary topological type.

The fourth modification is concerned with Procedure 2, the argument for recovering area loss at infinity. Now we also need to deal with holomorphic curves $\tilde{f}_{k}: D_{R_{k}}^{+} \rightarrow(M, J)$ with $R_{k} \rightarrow \infty\left(D_{R_{k}}^{+}=D_{R_{k}} \cap \bar{H}^{2}\right)$. The area loss $a_{\infty}$ of $\left\{\tilde{f}_{k}\right\}$ at $\infty$ is defined in the same way as (4.9), with $D_{R_{k}}, D_{R}$ replaced by $D_{R_{k}}^{+}, D_{R}^{+}$. The neck limit $l_{\infty}$ of $\left\{\tilde{f}_{k}\right\}$ is defined as (4.14) with $\partial D_{R}$ replaced by $\partial D_{R}^{+} \backslash \mathbb{R}$. Then it is clear that Procedure 2 carries over, if use of Theorem 3.3 is replaced by that of Theorem 3.4. Finally, degenerating conformal structures can be treated by doubling the Riemann surfaces. Only minor modification is needed to treat collars about shrinking geodesics which are originally boundary circles.

\section{REFERENCES}

[El] Y. Eliashberg, Filling by holomorphic discs and its applications, preprint, Stanford, 1990.

[Fe] H. Federer, Geometric measure theory, Springer, New York, 1969.

[Ga] L. Z. Gao, Convergence of Riemannian manifolds, Ricci pinching and $L^{N / 2}$-curvature pinching, preprint.

[Gi] M. Giaquinta, Multiple integrals in the calculus of variations and nonlinear elliptic systems, Princeton Univ. Press, Princeton, NJ, 1983.

[Gro] M. Gromov, Pseudo holomorphic curves in symplectic manifolds, Invent. Math. 82 (1985), 307-347.

[Grü] M. Grüter, Regularity of weak H-surfaces, J. Reine Angew. Math. 329 (1981), 1-15.

[Grü-Hi-Ni] M. Grüter, S. Hildebrandt and J. C. C. Nitsche, On the boundary behaviour of minimai surfaces with a free boundary which are not minima of the area, Manuscripta Math. 35 (1981), 387-410.

[Ha-Wi] P. Hartman and A. Wintner, On the local behavior of solutions of nonparabolic partial differential equations, Amer. J. Math. 75 (1953), 449-476.

[Hi] S. Hildebrandt, Nonlinear elliptic systems and harmonic mappings, Proc. Beijing Symp. Diff. Geom. \& Diff. Eq. 1980, Science Press, Beijing, 1982.

[Ho-Spru] D. Hoffman and J. Spruck, Sobolev and isoperimetric inequalities for submanifolds in Riemannian manifolds, Comm. Pure Appl. Math., 1970.

[Jo1] J. Jost, Two-dimensional geometric variational problems, Wiley-Interscience, New York, 1991.

[Jo2] - On the regularity of minimal surfaces with free boundaries in Riemannian manifolds, Manuscripta Math. 56 (1986), 279-291.

[Jo3] _ Continuity of minimal surfaces with piecewise smooth free boundaries, Math. Ann. 276 (1987), 599-614.

[Mc] D. McDuff, Elliptic methods in symplectic geometry, Bull. Amer. Math. Soc. 23 (1990).

[Oh] Y.-G. Oh, Removal of boundary singularities of pseudo-holomorphic curves with Lagrangian boundary conditions, preprint NYU, 1990.

[Pan] P. Pansu, Pseudo-holomorphic curves in symplectic manifolds, preprint, Ecole Polytechnique, Palaiseau, 1986.

[Par-Wo] T. H. Parker and J. G. Wolfson, A compactness theorem for Gromov's moduli space, preprint, 1991.

[Sa-U] J. Sacks and K. Uhlenbeck, The existence of minimal immersions of 2-spheres, Ann. of Math. (2) 113 (1981), 1-24. 
[Sc] R. Schoen, Analytic aspects of the harmonic map problem, Lectures on Partial Differential Equations (S. S. Chern, ed.), Math. Sci. Res. Inst. Publ. Ser., Springer, Berlin, 1984.

[Wo] J. G. Wolfson, Gromov's compactness of pseudo-holomorphic curves and symplectic geometry, J. Differential Geom. 28 (1988), 383-405.

[Ye1] R. Ye, Regularity of a minimal surface at its free boundary, Math. Z. 198 (1988), 261-275.

[Ye2] _ On minimal surfaces of higher topology, preprint, Stanford, 1988.

[Ye3] _ An isoperimetric inequality for Riemannian submanifolds with free boundary, in preparation.

[Ye4] _ Existence, regularity and finiteness of minimal surfaces with free boundary, preprint no. 1, SFB256, Bonn, 1987.

Department of Mathematics, University of California, Santa Barbara, California

E-mail address: yer@math.ucsb.edu 